\title{
Herramienta auditora sobre el acceso y preservación de la información audiovisual televisiva
}

\author{
Auditing tool on access and preservation of \\ television audiovisual information
}

\author{
Jorge CALDERA-SERRANO \\ Roberto Ovidio FREIRE-ANDINO²
}

\section{Resumen}

Se describe la herramienta auditora para determinar la correcta gestión, conservación y acceso a la información audiovisual en las cadenas de televisión. Dicha auditoría se presenta en formato de herramienta auditora que analiza una serie de parámetros, tales como: condiciones de accesibilidad, estado de conservación, recursos humanos y su formación, recursos económicos a disposición del servicio de gestión documental, análisis de los espacios físicos, descripción y evaluación de software y hardware, condiciones físicas de conservación, descripción documental realizada por el servicio de documentación y la identificación de la existencia de políticas de preservación, tratamiento y conservación de información en las empresas audiovisuales. Todo ello, para determinar los puntos de mejora para una correcta conservación del patrimonio audiovisual de la cadena y del Patrimonio Nacional Audiovisual de un país.

Palabras clave: Accesibilidad. Auditoría de la información. Empresas televisivas. Gestión de la información audiovisual. Información audiovisual. Preservación documental.

\begin{abstract}
This paper aims at describing the audit tool that can determine the correct management, preservation and access to audiovisual information within television networks. The audit is presented in the format of an auditing tool that analyzes a number of parameters, such as accessibility, state of preservation, human resources and training, economic funds which are available for the document management services, analysis of physical spaces, description and evaluation of software and hardware, physical conditions of storage, document description given by the documentation service and identification of existing policies for preservation, processing and storage of information in audiovisual companies. All this is done to determine which areas can be improved in order to offer proper preservation of the audiovisual heritage of not only television networks, but also the National Audiovisual Heritage of a country.
\end{abstract}

Keywords: Accessibility. Information audit. Television companies. Audio-visual information management. Audiovisual information. Documentary preservation.

\section{Introducción}

El trabajo que se presenta cuenta con la peculiaridad de ser una herramienta auditora gestada en el marco de un proyecto de investigación de la Secretaría Nacional de Educación Superior, Ciencia, Tecnología e Innovación (Senescyt) del Gobierno de la República del Ecuador. No obstante, la ventaja de dicha herramienta

\footnotetext{
1 Universidad de Extremadura, Facultad de Ciencias de la Documentación y la Comunicación, Departamento de Información y Comunicación. Plazuela de Ibn Marwan, s./n., 06071, Badajoz, España. Correspondencia a nombre de/Correspondence to: J. CALDERA-SERRANO.E-mail: <jcalser@alcazaba.unex.es>.

2 Universidad Central del Ecuador, Facultad de Comunicación Social, Carrera Comunicación Social. Quito, Ecuador.

Recebido el 24/11/2014, re-presentado el 19/2/2015 y aceptado para su publicación el 1/9/2015.
} 
es su validez para la evaluación de cualquier cadena de televisión, independientemente del país u origen.

Se desea llegar a obtener una visión de conjunto de cómo se encuentra la información audiovisual de las cadenas. Esta visión es igualmente útil para determinar la situación en la que se encuentran elementos específicos del sistema que integra la gestión, acceso y conservación del material audiovisual que almacenan e, hipotéticamente, custodian las cadenas de televisión.

A continuación se expone la herramienta utilizada para la evaluación de la colección audiovisual de las cadenas televisivas. Como podrá apreciarse tras su análisis, la herramienta auditora cuenta con diferentes epígrafes, pero todos siempre teniendo muy presente el fin último del análisis: la valoración del acceso y conservación del Patrimonio Audiovisual. Por lo tanto, dicha valoración será una buena herramienta de mejora tanto como para las cadenas como para la potencial implementación de políticas gubernamentales en materia de conservación y salvaguarda del patrimonio audiovisual.

Los puntos que a continuación se desarrollan tratan diferentes aristas en el marco de las colecciones audiovisuales, analizando tanto su accesibilidad como su descripción documental y su patrimonio así como alcance y comercialización. Es evidente que las consecuencias de una correcta conservación de esta información se transformará en la posibilidad de accesibilidad, y una correcta conservación documental se transformará en una mejor calidad del material al cual se tenga acceso (Edmondson, 2004).

Hemos de señalar previamente que los sistemas de información en las organizaciones televisivas son muy complejos (Caldera-Serrano \& Arranz-Escacha, 2012). No obstante, sí que hemos de tener muy claro que dichos sistemas cuentan con obligaciones muy diferentes en función de si su titularidad es de carácter público o privado. La diferencia es sustancial, derivado de las posibilidades de las políticas y líneas de actuación gubernamentales que pueden ser ejercidas sobre uno u otro tipo de empresas. Las empresas privadas sólo tienen que rendir cuenta a su accionariado, mientras que las empresas públicas audiovisuales tienen la obligación de la conservación eficiente del patrimonio generado. Esta dualidad en los objetos analizados por la herramienta que posteriormente se describe va a estar presente en todo momento, en especial en su adaptación y uso a los medios y, por lo tanto, a las potenciales recomendaciones que se realicen a la vista de los resultados obtenidos.

Se ha de incidir muy claramente en la dificultad que tiene el investigador y mucho más el ciudadano en poder acceder a los contenidos de los archivos audiovisuales. Independientemente de su titularidad pública o privada, los medios de comunicación son especialmente reacios a facilitar el acceso a los contenidos audiovisuales (Hidalgo Goyanes, 2013).

\section{Procedimientos metodológicos}

Tal y como se ha señalado, el trabajo se encuadra en un proyecto de investigación mucho más ambicioso como es el análisis del estado de conservación y accesibilidad a la información audiovisual por parte de las cadenas tanto públicas como privadas de la República del Ecuador. Para conseguir alcanzar este objetivo se ha tenido que llevar a cabo un estudio previo en pos de determinar las variables que deben tenerse en cuenta con el fin de analizar la situación real de estos archivos audiovisuales.

Para desarrollar esta herramienta se han seguido cinco fases:

- Fase 1: Revisión bibliográfica;

- Fase 2: Visita a centros audiovisuales y archivos de televisión;

- Fase 3: Redacción de herramienta;

- Fase 4: Evaluación de resultado (revisión de experto);

- Fase 5: Retroalimentación y redacción definitiva.

La implementación de las fases se ha llevado a cabo de forma paralela, aunque aquí se han presentado de forma secuencial. Cabe destacar la escasa bibliografía existente en materia de características de preservación de material audiovisual en los depósitos de televisión, derivado que las principales investigaciones suelen ser documentos internos generados por las propias cadenas de televisión. No obstante, existe cada vez más una creciente relación entre las empresas audiovisuales y el ámbito universitario y de la investigación, lo que facilita 
el intercambio de información y muy especialmente de experiencias, lo cual es sin lugar a dudas muy enriquecedor para ambas partes. En Brasil destacamos la labor del grupo de investigación coordinado por la Prof. Dra. Miriam Paula Manini a cargo del proyecto "Documentos audiovisuais, informação e memória: identificação de acervos fotográficos e fílmicos no Distrito Federal", en la Faculdade de Ciência da Informação de la Universidade de Brasília.

Aunque es escaso el material específico existente sobre la auditoría de la información audiovisual en televisión, sí existe un número representativo de trabajos que versan sobre la conservación y digitalización de la documentación audiovisual en diferentes ámbitos de la sociedad. En este sentido, en beneficio de una lectura rápida y concisa y estimando pertinente el carácter acumulativo de la ciencia, se han utilizado más para la confección del cuadro de análisis que para la realización del estado de la cuestión y del arte en la materia en la que se centra el trabajo.

Pero si la revisión bibliográfica es básica como primer elemento en cualquier investigación científica, especialmente en el ámbito de las Ciencias Sociales, ha sido aún más importante la conservación y visitas a diferentes instituciones televisivas para analizar de primera mano la realidad empresarial y la forma acorde de gestión y conservación de estos soportes. Aunque existen políticas supranacionales y ayudas para la conservación de colecciones audiovisuales, las cadenas de televisión suelen contar tan sólo con sus propios recursos para la conservación de los mismos y, por lo tanto, es importante conocer la experiencias de éstos y las condiciones reales a las que se puede llegar a conservar el patrimonio audiovisual de la televisión y de la sociedad a la que sirve y de la que se sirve.

El contacto con estos profesionales y el análisis de la realidad nos ha ayudado a la confección real de la herramienta y ha determinado las fases posteriores como la de retroalimentación. Estos profesionales han sido encargados de evaluar el trabajo realizado y estudiar la viabilidad de dicha herramienta.

Por lo tanto, el objetivo principal propuesto para el estudio presente es la creación de una herramienta auditora que muestre la realidad de la conservación patrimonial audiovisual y el acceso a la información por parte de la sociedad en general y de los periodistas de la cadena en particular. En ningún caso está planteada para comparar televisiones, su gestión o accesibilidad, sino para conocer el estado actual y la elaboración de la foto fija que nos describa el cómo se realiza la labor, en qué elementos puede mejorarse y en qué debe trabajarse para garantizar la preservación del patrimonio audiovisual de un país, que, volvemos a recordar, en el caso de nuestro trabajo se ha centrado en la República del Ecuador. Trabajo importante previo es el realizado por la Associação Portuguesa de Bibliotecários, Arquivistas e Documentalistas (2012), quienes han realizado un diagnóstico de la preservación del Patrimonio Audiovisual de Portugal.

Otros objetivos específicos que ayudan a alcanzar el objetivo general descrito con anterioridad serían:

a) Identificación de los contenidos (cobertura temática y alcance) del archivo televisivo de la cadena;

b) Descripciónyanálisis de las políticas institucionales en materia de conservación y preservación del material audiovisual, evaluando su adecuación a los requerimientos de la conservación patrimonial;

c) Evaluación de los Recursos Humanos para llevar a cabo una correcta descripción, almacenamiento, conservación y difusión de recursos audiovisuales televisivos;

d) Señalización de las características físicas de los espacios contenedores de los archivos televisivos y su adecuación a las necesidades de conservación del material audiovisual, atendiendo a su naturaleza fotográfica, sonora, audiovisual y a su realidad analógica o digital;

e) Descripción de software de gestión de la información audiovisual en su sistema de gestión de información y documentación audiovisual;

f) Estudio y determinación de los elementos que posibilitan e impiden el acceso ágil a la información audiovisual de las empresas televisivas;

g) Identificar la descripción documental de la información audiovisual para así detectar los posibles errores y material perdido por mala praxis en las colecciones audiovisuales televisivas;

h) Identificar las condiciones actuales de preservación y conservación de la información 
audiovisual, identificando posibles líneas de restauración, preservación y mejora si así se estimase necesario.

\section{La auditoría de la información}

Las diferentes entidades y organizaciones han implementado en las últimas décadas mecanismos para mantenerse a la vanguardia con el fin de satisfacer los requerimientos de sus usuarios. Entre estas herramientas se han desarrollado desde aquellas que tienen relación con la reestructuración del negocio hasta elementos para la validación de variables de los sistemas. Pero, sin lugar a dudas, se han puesto en marcha mecanismos de evaluación, seguimiento y medición que permiten a las empresas "conocer sus debilidades, fortalezas, oportunidades y amenazas, para así desarrollar estrategias de mejoramiento" (Gutiérrez Garzón, 2003, p.14).

En el ámbito de la gestión del conocimiento, hace ya décadas que se están llevando a cabo las denominadas auditorías de la información, para poder detectar las ventajas e inconvenientes con los que cuentan los servicios de información que llevan a cabo dicha gestión del conocimiento, la información y la documentación. Para ello, y una vez auditados los servicios de información, se podrán generar sinergias, mejoras e implementación de planes de actuación para mejor uso y optimización de los servicios documentales.

La auditoría podrá analizarse desde diversos puntos de vista. No obstante, deberá quedar fijada en todo el ciclo de vida de los documentos, y potenciando para cierto tipos documentales y, con ciertas características, en su accesibilidad y conservación.

\section{Auditando}

Las definiciones que existen sobre auditoría son varias y dispersas. Mientras unas se centran en la gestión documental de la información así como en los sistemas de información que lo sustentan, otras organizan los procesos de la información, el flujo informacional, etc. (Buchanan \& Gibb, 1998).

Las auditorías suelen utilizar el conocimiento de expertos para la validación y análisis de los sistemas, entre ellas el documental. Así, estos expertos detectan los errores en relación con la gestión, diseño, implementación, etc. de los sistemas de información. La auditoría aplicada a los sistemas de gestión puede definirse como

[...] un examen sistemático, planeado y organizado que determina si las actividades y los resultados relacionados con la gestión de documentos cumple con las disposiciones establecidas (métodos, procedimientos, etc.), y si éstas se aplican en forma efectiva para alcanzar los objetivos planteados, no sólo por la unidad responsable de la gestión documental, sino por la organización (Gutiérrez Garzón, 2003, p.17).

Por lo tanto, y atendiendo a lo señalado anteriormente por Gutiérrez Garzón, se definen tres tipos de auditorías de gestión documental: la primera de ellas es la que relaciona la gestión de la información en el marco de la organización; la segunda la que analiza la existencia y el cumplimiento de políticas y procedimientos para llevar a cabo de forma correcta y consensuada la gestión documental de la información; y la tercera y última es la inclusión de la información en el marco de la organización para generar el menor ruido y silencio a tenor de las necesidades reales y potenciales de la entidad a la cual se debe el servicio de documentación.

La auditoría debe contestar a innumerables cuestiones. Todas ellas se plantean atendiendo a los parámetros anteriormente mencionados: necesidades informativas, accesibilidad a dicha información, adecuación de la organización, integración del sistema en el marco organizativo, da respuesta a las consultas planteadas por los usuarios, etc.

Gutiérrez Garzón (2003) señala los principales resultados posibles que pueden lograrse en una auditoría de la gestión de la información documental en una organización empresarial o en entidad de carácter público:

1) Balance de los procesos y actividades de la gestión de documentos en la organización;

2) Identificación de puntos críticos y cuellos de botella, lo que permite establecer las acciones de mejora para minimizarlos;

3) Se da prioridad a las áreas con problemas y los problemas como tal; 
4) Permite el diseño de distintos mapas documentales;

5) Permite la identificación de expertos en temas puntuales;

6) Verificación del cumplimiento de los requerimientos normativos y legales;

7) Identificación de las necesidades y expectativas de la organización, en lo relacionado con la gestión de documentos;

8) Establecimiento de estrategias inherentes a la gestión de documentos, por ejemplo, implementación de tecnologías de información, bases de datos, etc.;

9) Elaboración del plan de acción y proyectos relacionados con la gestión de documentos.

Igualmente señala los beneficios que se podrían obtener, los cuales son:

1) Reconocer los principales obstáculos que tiene la gestión de la información, así como reconocimiento y determinación del sobrecoste para la entidad por conceptos de mala organización;

2) Evaluación de gestión de los documentos respeto a las normativas nacionales e internacionales de gestión;

3) Entender los flujos informacionales tanto dentro de la organización como aquellos que pudieran salir fuera del propio sistema interno empresarial;

4) Identificar las necesidades reales de usuarios para así facilitar en forma y tiempo los documentos que potencialmente puedan requerir los usuários;
5) Mostrar a la organización las potencialidades del Sistema de información en el ámbito de la organización.

El procedimiento para llevar a cabo la auditoría debe reunir evidencias para su análisis y lograr así los objetivos planteados, dominando claramente el ámbito de trabajo en el que se enmarca la organización. Para poder auditar se debe conocer perfectamente la organización en concreto y la relación con su entorno.

La fase de ejecución del proyecto auditor debe recopilar las evidencias, de la manera señalada, así como la revisión y análisis de las muestras recogidas. Finalmente se deberá redactar el informe auditor con el cual se plasmen las líneas de mejora de la gestión de la información en la organización. Es fundamental el análisis de diferentes elementos, como la propia gestión, el software, los recursos humanos, las herramientas documentales, las políticas que conforman las directrices y normas del servicio de información, etc.

Todo este proceso tiene como fin último no sólo la confección de un informe final, sino muy especialmente un proceso de retroalimentación con la organización. Ello busca mostrar las líneas de mejoras ponderadas y organizadas de forma secuencial siempre que sea posible.

\section{Resultados}

Identificación de la denominación y ubicación de la cadena. Igualmente importante es la persona de contacto con la empresa que, en definitiva, será el

Cuadro 1. Los elementos que conforman esta herramienta son los siguientes.

\begin{tabular}{ll}
\hline Identificación & Contenidos \\
\hline Nombre de la cadena & Horas de emisión \\
Tipo de televisión: Temática Generalista & Horas de archivo/total del archivo \\
Titularidad: Pública Privada & Horas programas de entretenimiento \\
Sede principal & Horas programas informativos \\
Otras sedes & Tipos de contenidos \\
Definición de la propia empresa & \\
Responsable Doc. & \\
Teléfono & \\
Correo electrónico & \\
Url:// & \\
\hline
\end{tabular}

Fuente: Elaboración propia (2015). 
encargado de coordinar de forma interna la auditoría de la documentación. Si bien se requiere que esta persona de contacto sea el responsable del servicio de documentación, este deberá estar determinado por la propia cadena televisiva (Cuadro 1).

En este apartado se intenta obtener una visión general tanto de la importancia de la colección como de su contenido en horas. Al ser todas las televisiones analizadas semejantes en sus fines, el contenido temático en sí suele ser equivalente o muy parecido en cada una de estas empresas. Por ello, se desea conocer el volumen de documentos/horas de imágenes en movimiento con lo que cuenta la cadena.

La determinación e identificación unívoca de políticas en el marco de los sistemas de gestión documental marcan claramente las pautas y líneas a seguir por los profesionales de la documentación, al igual que nos definen cómo se quiere alcanzar los objetivos del servicio y cuáles son estos principios y estos objetivos (Caldera-Serrano, 1997). Aunque existirá un contacto con los profesionales para determinar el grado de cumplimiento de estas políticas, es indispensable que existan estas guías con el fin de que todo el equipo trabaje en la misma línea y que, por lo tanto, los objetivos y métodos de trabajo sean compartidos. Dichas políticas de los servicios de documentación deben venir

Cuadro 2. Políticas del Servicio de Información.

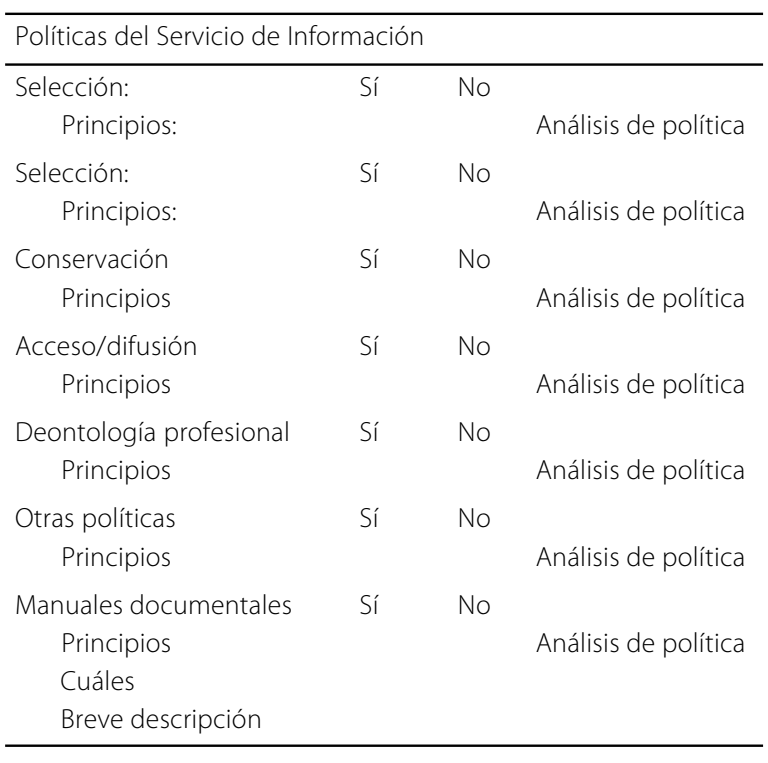

Fuente: Elaboración propia (2015). acompañadas por políticas estatales de acceso a los contenidos (Salvador Benítez, 2010) (Cuadro 2).

En este apartado se analizarán los recursos humanos propios del sistema documental, ya sean propiedad de la cadena misma o hayan sido externalizados por la empresa. Es indispensable conocer elementos que definan las capacidades y destrezas tanto de los responsables como de los operariosdocumentalistas del Servicio (Cuadro 3).

En este apartado se analizan tanto los lugares disponibles para las colecciones como los espacios físicos para la correcta gestión documental; se examinará en el epígrafe la herramienta informática de gestión documental. El análisis computacional es básico para determinar las potencialidades del sistema. Un mal sistema informático genera graves problemas para la correcta gestión de la documentación generada en la cadena $y$, por ello, se analiza si sigue los criterios documentales establecidos por el personal de la cadena, además de analizar si cuenta con posibilidades no explotadas por el personal del Servicio de Información (Cuadro 3).

Sin lugar a dudas, las cuestiones relativas a la accesibilidad son muy importantes debido al carácter interno que tiene la documentación en las empresas audiovisuales. Los archivos de televisión existen ya que son útiles a la propia empresa, y por lo tanto se debe garantizar el acceso a la información por parte del personal de la propia cadena de forma rápida y segura. Ahora bien, dicha accesibilidad debe garantizar igualmente la integridad física del soporte; es decir, que un material que se conforma como el Patrimonio Histórico Audiovisual de una región o un país se conserva en las mejores condiciones (Cuadro 3).

El acceso a los materiales en el marco de la propia empresa también debería garantizar la accesibilidad por parte de otros miembros de la comunidad, para así localizar, utilizar y verificar la información para usos posteriores muy distintos para los que fueron creados en su origen. Se debe garantizar la integridad documental (Caldera-Serrano \& Zapico-Alonso, 2004) del soporte pero también el acceso a los miembros de la propia cadena y, en caso de televisiones públicas, a individuos que realicen labores de investigación en diversas materias.

El control de la gestión documental es fundamental para el correcto funcionamiento de un 
Cuadro 3. Recursos humanos.

Recursos humanos

Número total de RR.HH. Servicio de Documentación

Porcentaje sobre el total de la plantilla

Organigrama RR.HH. Del Servicio de Documentación

Número de persona por Secciones

Titulación de los empleados

¿Plan de formación de empleados del Servicio de Documentación?

En caso positivo, explicar brevemente cuál es el plan y en qué consiste

Experiencia (en meses) en el Servicio de Documentación

Entrevista con plantilla: Situación de la conservación y la gestión documental

\begin{tabular}{l}
\hline Espacios físicos \\
\hline RR.HH. ¿Espacio físico propio para servicio? (descripción) \\
RR.HH. ¿Espacio físico propio para análisis/difusión? \\
RR.HH. Condiciones de los puestos de trabajo ¿individual o compartido? ¿equipamiento? \\
RR.HH. ¿Ergonomía correcta del puesto de trabajo? \\
Colección. ¿Espacio físico propio para la instalación de la colección? \\
Colección. ¿Separación entre tipos documentales? \\
Colección. ¿Separación entre formatos audiovisuales? \\
Colección. ¿Fácilmente accesible para los usuarios? \\
\hline
\end{tabular}

Software propio/adquirido

Implementado con criterios documentales

Módulos y descripción de módulos

Herramientas de descripción

Análisis de la herramienta atendiendo a potencialidades del sistema

Análisis de la herramienta atendiendo al uso de los profesionales

Accesibilidad al material en la cadena

Niveles de accesibilidad en la empresa

Control de acceso y uso de los contenidos

Control de los soportes físicos

Venta de material

Costes del material

Acceso especiales

Fuente: Elaboración propia (2015).

Nota: RR.HH.: Recursos Humanos.

sistema de información documental. Este apartado, al cual se le dedica mayor peso en el análisis auditor, desea detectar los posibles errores y vicios de ese sistema, intentando así plasmarlos y clarificarlos para poder posteriormente resolverlos, siempre y cuando dicha posibilidad sea optimizada por la empresa.

La gestión documental de información audiovisual televisiva es, sin lugar a dudas, un tema complejo derivado de la naturaleza audiovisual del material (Nuño Moral \& Caldera-Serrano, 2000), siendo necesario analizar tanto elementos visualizados como aquellos referenciados en la banda sonora y, por supuesto, detectar aquellos mensajes resultados de la unión de la banda imagen y el sonido. Todo ello teniendo presente la necesidad de contar con campos tanto en texto libre como en lenguaje documental controlado (Cuadro 4).

Se va a analizar un elemento clave para el acceso al Patrimonio Nacional Audiovisual: la preservación física de los soportes documentalesque albergan la información. En este epígrafe se analizarán los aspectos relativos a la preservación y conservación de los diferentes materiales audiovisuales, ya sean analógicos o digitales (Caldera- 
Cuadro 4. Descripción documental.

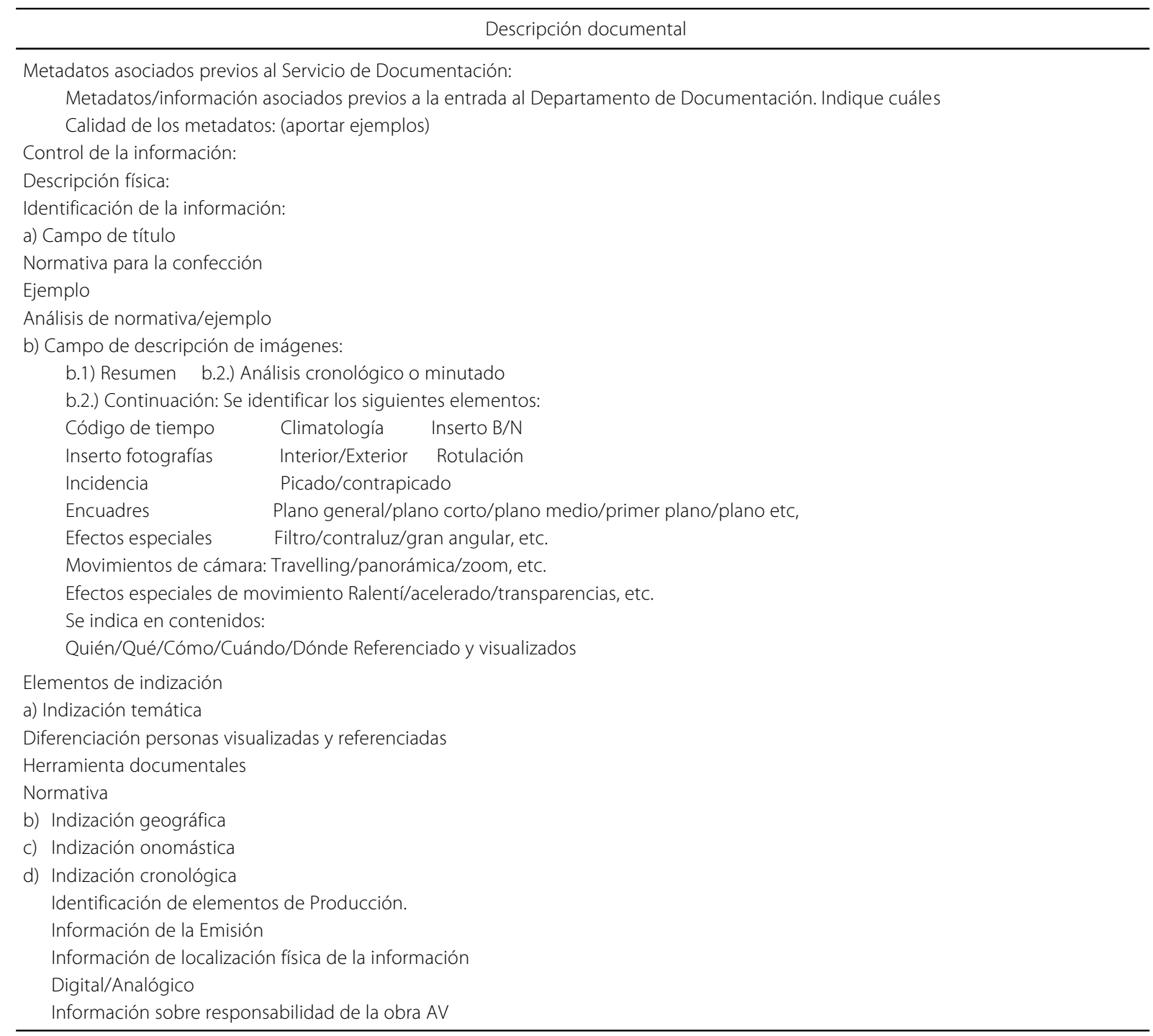

Fuente: Elaboración propia (2015).

Notas: AV: Audiovisual; B/N: Blanco y negro.

Serrano, 2008; Saavedra Bendito, 2011), o incluso albergando la posibilidad de contar con material cinematográfico (González-Ruiz et al., 2012) (Cuadro 5).

Identificar que no existe una mejor manera de llevar a cabo políticas de conservación que mediante implementaciones de tareas de digitalización, tanto en la producción y emisión como también en la preservación documental. La facilidad de clonar y duplicar contenidos en formato digital facilita la preservación en distintos lugares y garantiza la integridad de los contenidos. Parece, a día de hoy, que la perdurabilidad de los contenidos audiovisuales está asegurada, ya que en caso de aparición de nuevos formatos de lectura de los contenidos, siempre se podrá volcar a los nuevos formatos.

\section{Conclusión}

El trabajo expuesto no tiene la finalidad de comparar ni de castigar, sino la de analizar y ayudar a mejorar la preservación, conservación y acceso a la información. Por ello, se deben analizar elementos tales como las condiciones físicas, el tratamiento documental - que garantiza su accesibilidad entre la cantidad de horas 
Cuadro 5. Conservación de las colecciones audiovisuales.

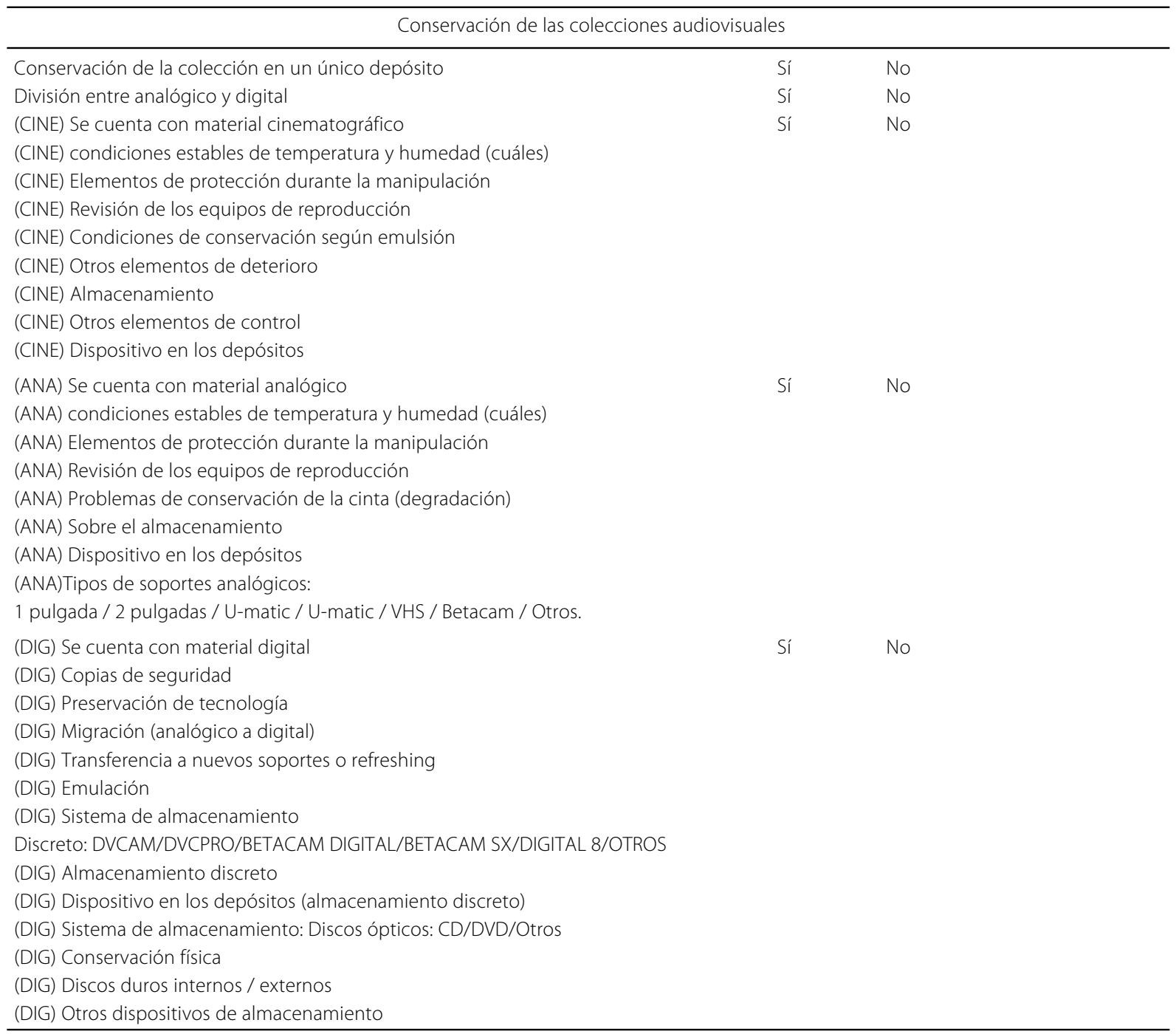

Fuente: Elaboración propia (2015).

Notas: ANA: Analógico; CINE: Cinematográfico; DIG: Digital.

y horas de emisión y brutos de la cadena -, los recursos económicos, humanos, espaciales, etc. Todos estos son todos elementos que nos ayudan a mostrar una imagen eficaz y real de la situación de las cadenas.

No se presenta una cuantificación en los parámetros evaluados con el fin de determinar hasta qué punto se cumplen normativas o dejan de llevarse a cabo. En su lugar se propone un análisis en conjunto para determinar cuáles son los elementos que deben mejorarse y, sobre todo, para conocer en qué estado se encuentran las colecciones audiovisuales.
Suele ser relativamente fácil determinar cuándo una colección está en peligro, dónde su durabilidad y preservación futura no está garantizada. Sin embargo, entre la dejadez más absoluta y la imposible perfección existen muchas escales con posibilidades de mejora, y es aquí donde se entiende importante una revisión externa de las cadenas para poder identificar vicios y maneras incorrectas de trabajo dadas por válidas.

Se audita para mejorar, se evalúa para conocer en qué estado se encuentra la información audiovisual de la empresa televisiva, y se describe para poder crear 
planes de actuación para llevar a cabo de manera más correcta las labores encomendadas a los servicio de gestión documental de las empresas televisivas. Y todo ello partiendo de una premisa fundamental, y es que la conservación, preservación y accesibilidad a los documentos audiovisuales debe estar garantizada. Y debe estarlo porque la información audiovisual es memoria colectiva viva, es parte de la sociedad por medio de la cual se ha generado, es nuestro pasado más reciente y más remoto, y debe garantizarse su conservación al igual que se garantiza la preservación de información bibliográfica (bibliotecas nacionales), de información archivística administrativa (archivos históricos nacionales), de colecciones audiovisuales cinematográficas (cinematecas o filmotecas nacionales), etc. El material que se encuentra en los archivos de televisión es de toda la sociedad, de todo el país, siendo especialmente importante contar con dicha cultura de la preservación en las televisiones públicas.

\section{Referencias}

Associaçao Portuguesa de Bibliotecários, Arquivistas e Documentalistas. Os arquivos audiovisuis em Portugal: un diagnóstico. Lisboa: Associaçao Portuguesa de Bibliotecários, Arquivistas e Documentalistas, 2012. Disponível em: <http:// www.bad.pt/publicacoes/diagnostico_patrimonio_ audiovisual_nacional. pdf>. Acesso em: 16 fev. 2015.

Buchanan, S.; Gibb, F. The information audit: An integrated strategic approach. International Journal of Information Management, v.18, n.1, p.29-47, 1998.

Caldera-Serrano, J. La red de información juvenil de la Comunidad Autónoma de Extremadura. In: Jornadas Andaluzas de Documentación, 1., 1997, Sevilla. Anales.. Sevilla: Jadoc, 1997. p.111-117.

Caldera-Serrano, J. Changes in the management of information in audio-visual archives following digitization: Current and future outlook. Journal of Librarianship and Information Science, v.40, n.1, p.13-20, 2008.

Caldera-Serrano, J.; Arranz-Escacha, P. Documentación audiovisual en televisión. Barcelona: EPI-UOC, 2012.

Caldera-Serrano, J.; Zapico-Alonso, F. La fórmula de comunicación de Lasswell como método para implementar bases de datos documentales en los medios audiovisuales. Investigación Bibliotecológica, v.18, n.37, p.110-131, 2004.
Preservar hoy es garantizar el acceso a la información futura. Este acceso debe estar regulado y asegurado por medio de políticas institucionales en el marco de las cadenas de televisión y por políticas gubernamentales, si así se determinara necesario.

\section{Reconocimientos}

Este trabajo ha sido financiado por el Gobierno de Extremadura (Consejería de Educación, Ciencia y Tecnología) y el Fondo Social Europeo dentro del plan de apoyo a las actuaciones de los Grupos de Investigación inscritos en el catálogo de la Junta de Extremadura (GR10019).

\section{Colaboración}

Todos los autores contribuyeron en todas las etapas del artículo.
Edmondson, R. Filosofía y principios de los archivo audiovisuales. París: Unesco, 2004. Disponible en: <http://unesdoc.unesco. org/images/0013/001364/136477s.pdf>. Acceso en: 16 feb. 2015.

González-Ruiz, D.; Térmens, M.; Ribera, M. Aspectos técnicos de la digitalización de fondos documentales. El Profesional de la Información, v.21, n.5, p.62-70, 2012.

Gutiérrez Garzón, L. La auditoría de información como herramienta de evaluación y mejoramiento de la gestión de documentos. Biblios, n.16, p.14-22, 2003.

Hidalgo Goyanes, P. Prevenir la amnesia colectiva: el acceso público a los archivos de televisión. Documentación de las Ciencias de la Información, v.36, p.143-166, 2013.

Nuño Moral, M.V.; Caldera-Serrano, J. Etapas del tratamiento documental de imagen en movimiento para televisión. Revista General de Información y Documentación, v.12, n.2, p.375-392, 2000.

Saavedra Bendito, P. Los documentos audiovisuales, ¿qué son y cómo se tratan? Gijón: Trea, 2011.

Salvador Benítez, A. Políticas de salvaguardia y acceso en los archivos audiovisuales de televisión: marco jurídico y nuevos servicios interactivos en la televisión digital. Derecom, n.2, p.1-18, 2010. 